\title{
HYPER-HEURISTIC APPROACH FOR IMPROVING MARKER EFFICIENCY
}

\author{
Daniel Domović1, Tomislav Rolich¹, Marin Golub²
}

${ }^{1}$ University of Zagreb, Faculty of Textile Technology, Department of Fundamental, Natural and Technical Sciences, Prilaz baruna Filipovića $28 a, 10000$

Zagreb, Croatia, tel.:+385 13712 545, fax: + 38513712 599, mailing addresses: \{daniel.domovic@ttf.hr, tomislav.rolich@ttf.hr\}

${ }^{2}$ University of Zagreb, Faculty of Electrical Engineering and Computing, Department of electronics, microelectronics, computer and intelligent systems, Unska 3, 10000 Zagreb, Croatia, tel.:+385 16129 502, fax: + 38516129 653, mailing address: marin.golub@fer.hr

Corresponding author e-mail: daniel.domovic@ttf.hr

\begin{abstract}
:
Marker planning is an optimization arrangement problem, where a set of cutting parts need to be placed on a thin paper without overlapping to create a marker - an exact diagram of cutting parts that will be cut from a single spread. An optimal marker that utilizes the length of textile material has to be obtained. The aim of this research was to develop novel algorithms for obtaining an efficient marker that would achieve competitive results and optimize the garment production in terms of improving the utilization of textile material. In this research, a novel Grid heuristic was introduced for obtaining a marker, alongside its improvement methods: Grid-BLP and Grid-Shaking. These heuristics were hybridized with genetic algorithm that determined the placement order of cutting parts using the newly introduced All Equal First (AEF) placement order. A novel individual representation for genetic algorithm was designed that was composed of order sequence, rotation detection and the choice of placement algorithm (hyperheuristic). Experiments were conducted to determine the best marker making method, and hyper-heuristic efficiency. The implementation and experiments were conducted in MATLAB using GEATbx toolbox on five datasets from the garment industry: ALBANO, DAGLI, MAO, MARQUES and MAN SHIRT. Marker efficiency in percentage was recorded with best results: $84.50 \%, 80.13 \%, 79.54 \%, 84.67 \%$ and $86.02 \%$ obtained for the datasets respectively. The most efficient heuristic was Grid-Shaking. Hyper-heuristic applied Grid-Shaking in $88 \%$ of times. The created algorithm is independent of cutting parts' shape. It can produce markers of arbitrary shape and is flexible in terms of expansion to new instances from the garment industry (leather nesting, avoiding damaged areas of material, marker making with materials with patterns).
\end{abstract}

\section{Keywords:}

Marker making, lay planning, production optimization, CAD/CAE, genetic algorithm, hyper-heuristic

\section{Introduction}

Marker planning is a critical arrangement process in the cutting room, in which a set of cutting parts needs to be placed on a thin paper of fixed width and arbitrary length. The product of such an arrangement is a marker, that is, an exact layout of cutting parts that will be cut from a single spread [1]. The aim of marker planning in garment manufacturing is to optimally allocate cutting parts on a marker in order to maximize the efficiency of material and to reduce the amount of waste, since the cost of textile material may constitute even half of the final garment cost [2]. When planning a marker, it is necessary to respect the placement rules, which ensure the most rational material consumption. The professional limitations that need to be taken into account when planning a marker include: the shape of cutting parts, material width, direction of warp and weft, patterns on material and so on [3].

Based on the number of cutting parts in a marker, half-garment and whole garment markers are distinguished. Half-garment markers are composed of half of cutting parts for a particular garment item. Whole-garment marker is composed of all cutting parts for a particular garment. Most commonly, the area of waste in the whole-garment markers is smaller than in halfgarment markers. Markers can also be distinguished based on the number of sizes they are composed of. Thus, a single size and multi-size markers are distinguished. Single size markers are composed of cutting parts of a single garment size. Multisize markers can be composed of cutting parts of multiple same size garments, or of multiple different size garments. Additionally, based on a position of a cutting part in a marker, multi-size markers can de diversified as: sectional (at least two garment sizes orthogonally placed one after the other), interlocking (at least two garment sizes non-orthogonally placed one after the other) or mixed multi-size (cutting parts are intermixed) [3].

In this research, a synergy between garment industry and computer science is created. Marker making is largely studied in computer science, where it is known as strip-packing problem or nesting $[4,5]$. It is formally defined as a combinatorial optimization problem, where a set of cutting parts $C P=\left\{c p_{1}\right.$, $\left.c p_{2}, \ldots, c p_{n}\right\}, n=|C P|$, need to be placed on a marker $M$, with arbitrary length and fixed width, so that the cutting parts $c p_{i}$ and $c p_{j}$ do not overlap $\left(c p_{i} \cap c p_{j}=0\right)$ and do not exceed the boundaries of a marker $\left(c p_{i} \cap M=c p_{i}\right)$. Cutting parts are 
defined as irregular polygons or items, while the marker is considered to be a rectangular shaped container [6]. Marker making is a subtype of packing problems. The taxonomy of packing problems has been presented in [5].

One of the first challenges a researcher faces while developing algorithms for automatic marker making is overlapping detection. Choosing a good overlapping detection model is important since the complexity and the execution time of a program depend on this implementation. The most common overlapping detection methods are: raster method, direct trigonometry, no-fit polygon (NFP) and constraint graphs. Raster method approximates both marker and cutting parts which influences the precision on the shape representation and increases memory usage $[7,8]$. These disadvantages are improved by direct trigonometry method. However, each change in the polygon position must be re-checked to see if the polygons overlap. Since a lot of calculations with decimal values need to be performed, it has an effect on execution time $[9,10]$. No-fit polygon is a popular method for finding an area where two polygons do not overlap. This method is faster than the direct trigonometry method, and operates on polygons without the need for approximation [11,12]. Constraint graphs are a set of acyclic graphs used to describe how items must be placed in relation to each other so that they do not overlap [6]. Using constraint graphs avoids overlapping.

In the literature, many approaches have been investigated for marker making. Some of the most efficient results have been obtained using the evolutionary algorithms, specifically with the genetic algorithm (GA, described in section 2.3). The genetic algorithm is a method for solving problems of search and optimization based on the nature's evolution principles. The genetic algorithm provides good solutions to the packing problem in combination with some local search algorithm. GA is used to determine the polygon layout, while local search technique is used for polygon placement. The local search algorithm is used to reconstruct the polygonal layout to minimize the amount of waste. In [13], GA was hybridized with quasi-Newton's local search method. GA has been applied on nesting irregular polygons based on the no-fit polygon and the lowest gravity center (LGC) in [8]. The advantage of this algorithm is that it successfully generates a polygon layout when the rotation of polygons is allowed and it solves the problem of inserting the polygon into waste space created between polygons. Different versions of bottom-left heuristics have been hybridized with GA in [14-16].

Placement order of cutting parts has a major influence on the marker efficiency. In GA, the order is defined by an individual represented as permutation. Simple heuristics have been investigated to determine the order of packaging [17]:

- For shelf algorithms: best width fit, worst width fit, best height fit, next fit, first fit, area fit

- For guillotine algorithms: best area fit, best short side fit, best long side fit, worst fit

- For maximal rectangles algorithms: bottom-left, best area fit, best short side fit, best long side fit

- For skyline heuristic: bottom-left, best-fit
Except for the genetic algorithm, other methods based on evolutionary computation, such as particle swarm optimization [18] and ant colony optimization [19] are also used in the literature. A heuristic approach hybridized with $(\mu+\lambda)$ evolution strategy is applied on shirts and swimsuits. A mixed-integer programming approach is described in [20]. Simulated annealing approach is described in [11].

Since it is often difficult to choose the most appropriate heuristic for a certain dataset, the need to approach solving the problem with several different heuristics has been imposed. Thus, a hyper-heuristic approach has been developed. By its initial definition in [21], a hyper-heuristic is a high level approach using which, a low-level heuristic can be selected from a set of implemented low-level heuristics and applied to a problem instance at a decision point. A taxonomy of hyper-heuristics has been presented in [22]. In [23], a hyper-heuristic based on genetic algorithm for solving strip-packing problem has been proposed. In [24], a hyper-heuristic has been developed as a high-level approach to choose between three hybrid metaheuristics: GA-LS (local search), GA-SA (simulated annealing) and GA-TS (tabu search). In [25], hyper-heuristic based on a messy genetic algorithm has been applied on a $2 D$ cutting stock problem.

Based on the literature overview, improvement possibilities have been identified. Analyzed algorithms are mostly specialized for solving a single type of packing problem. The largest number of algorithms are mainly used to create markers of rectangular shape and cannot easily be extended to create irregular markers (e.g., shape of leather). Also, not many solutions offer the ability to easily represent and to avoid possible damaged areas of a material (e.g., damaged part of leather). Also, most algorithms cannot be applied on materials with patterns. A lot of algorithms do not have the possibility to fill the waste space that might occur between two cutting parts.

Many authors use benchmark data sets used in the garment industry, obtained from [26] when executing and testing their algorithms. However, in some algorithms, the rotation of cutting parts in different directions is enabled (e.g., increments of $90^{\circ}$ angle, or even for an arbitrary angle of $0^{\circ}$ to $360^{\circ}$ ) to obtain better results. Rotation of cutting parts for an arbitrary angle may not be allowed for some textile materials since the direction of a warp of a cutting part has to be parallel with the length of a marker [3].

Shape of polygons is often approximated in order to lower the execution time and computational complexity. If irregular polygons are used, they are often approximated with the bounding box [27] or a convex hull [28] to create simplified polygons with smaller number of vertices to lower the computational complexity. The disadvantage of this approach is the large amount of waste that occurs between the edges of the described rectangle and the approximated polygon. Also, the precision of polygon visualization is reduced. Sometimes the authors employ algorithms for overlap detection prior to the algorithm execution, therefore decreasing the computation time. Since it is mostly done in the pre-processing phase, it is not even included in the time quota execution. 
Although commercial programs have been developed for marker making, their license is not affordable for small businesses.

Based on the identified improvement possibilities, the aim of the research was posed. A general and adaptable algorithm that could solve the problem of marker planning needs to be designed. The algorithm should be able to obtain a marker of arbitrary shape and must have the ability to operate on the cutting parts of arbitrary shape without the need for their approximation. It must have the ability to fill the waste space, if possible. Additionally, the algorithm must be easily extended to meet additional user needs such as the ability to determine material quality. The algorithm must achieve competitive results and have possible application in small businesses.

Therefore, a heuristic called Grid is introduced, alongside its two modifications Grid-BLP and Grid-Shaking. The main benefit of a Grid heuristic is its flexibility. The Grid heuristic is able to place any kind of regular or irregular polygon (cutting part) on any kind of material, whether it is a rectangular material, irregularly shaped leather or leather with defects or infeasible areas. Therefore, it can be applied on any type of dataset. Grid heuristic works with cutting parts directly - it does not approximate them, and therefore, preserves the cutting parts' representation.

Grid heuristics was hybridized with genetic algorithm. The main role of genetic algorithm is to guide the optimization process and obtain the placement order for cutting parts. In order to do so, a novel individual representation for the genetic algorithm has been designed. Prior, the algorithms mostly used permutation representation of an individual in GA to define the order in which the cutting parts will be placed on a marker. Individual representation used in this research is composed of three parts. Alongside the permutation part that defines the placement order, a rotation part has been added, that determines whether the cutting part should be rotated or not.

Hyper-heuristic has been defined in this research as the third parameter of an individual in GA that determines which of the three grid heuristics will be applied to reconstruct a placement from a defined order.

\section{Methodology}

\subsection{Grid heuristics}

A Grid heuristic is a raster-inspired 2D method for the placement of cutting parts on a marker. Inspiration method called the Dotted-board-model was described in [29]. In the most basic version of Grid heuristic, a set of points $\left(x_{i}, y_{i}\right)$ is created and equidistantly distributed alongside width and length of a marker in the container (Eq. 1-Eq..4). Value $d(\cdot)$ represents the distance between points.

$$
\begin{aligned}
& d\left(x_{i}-x_{i-1}\right)-d\left(x_{i+1}-x_{i}\right)=0 \\
& d\left(y_{i}-y_{i-1}\right)-d\left(y_{i+1}-y_{i}\right)=0
\end{aligned}
$$

$$
\begin{aligned}
& x_{i-1} \leq x_{i} \leq x_{i+1} \\
& y_{i-1} \leq y_{i} \leq y_{i+1}
\end{aligned}
$$

Cutting part is placed on a marker so that its reference point coincides with a grid point. It is then tested whether the cutting part exceeds the marker boundaries and whether it overlaps with the previously placed cutting parts. A point that satisfies these conditions is called a feasible point. Feasible points are being sought in the search direction from left to right, from bottom to top (Figure 1).

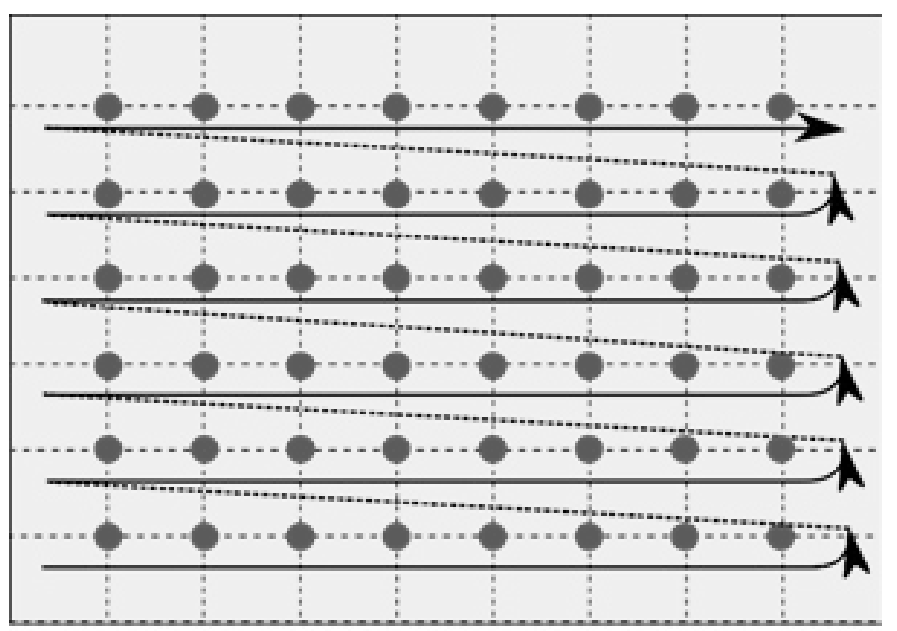

Figure 1. Empty grid and search direction

An example of placing the first nine cutting parts from a MAN SHIRT dataset using the Grid method is presented in Figure 2 (the numbers on the cutting parts indicate the order in which they were placed on a marker). One of the benefits of grid heuristics is that the waste space between cutting parts already placed on a marker can easily be filled if a cutting part of appropriate size is available (Figure $2 c-2 e$ ).

Improvements of the Grid heuristic have been developed in this research: Grid-BLP and Grid-Shaking. These heuristics combine the grid representation of a marker and a BLP or Shaking heuristics (methods inspired by the bottom-left heuristics) to tighten the placement of cutting parts and improve the marker efficiency.

Bottom-left is a placement heuristic. IIf an item $i$ needs to be placed in layout $L$, the bottom-left heuristics chooses the ultimate bottom and left possible position in $L$ for each item $i$ (i.e., a stable position) [6]. The position is obtained by iteratively sliding the item $i$ down and left, until a stable position in the layout $L$ is found. Such an arrangement can be achieved in the time complexity $\mathrm{O}\left(n^{2}\right)$, where $n$ is the number of polygons in a dataset [30]. Bottom-left heuristic is often used with metaheuristic algorithms [31].

If items to be placed on a marker are irregular (e.g., cutting parts), sliding algorithms based on rectangles (BL BLLT and BLD) and polygons (BLP, BLDP, BLF) can be used [32].

Sliding algorithm based on polygons called BLP is used in this paper and hybridized with Grid heuristic to create Grid- 


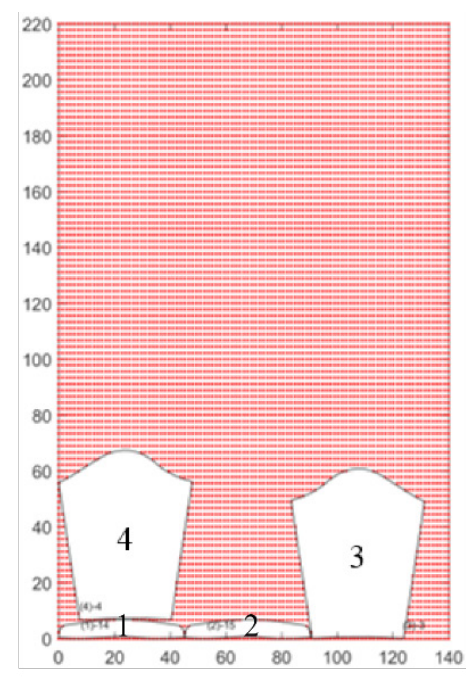

a
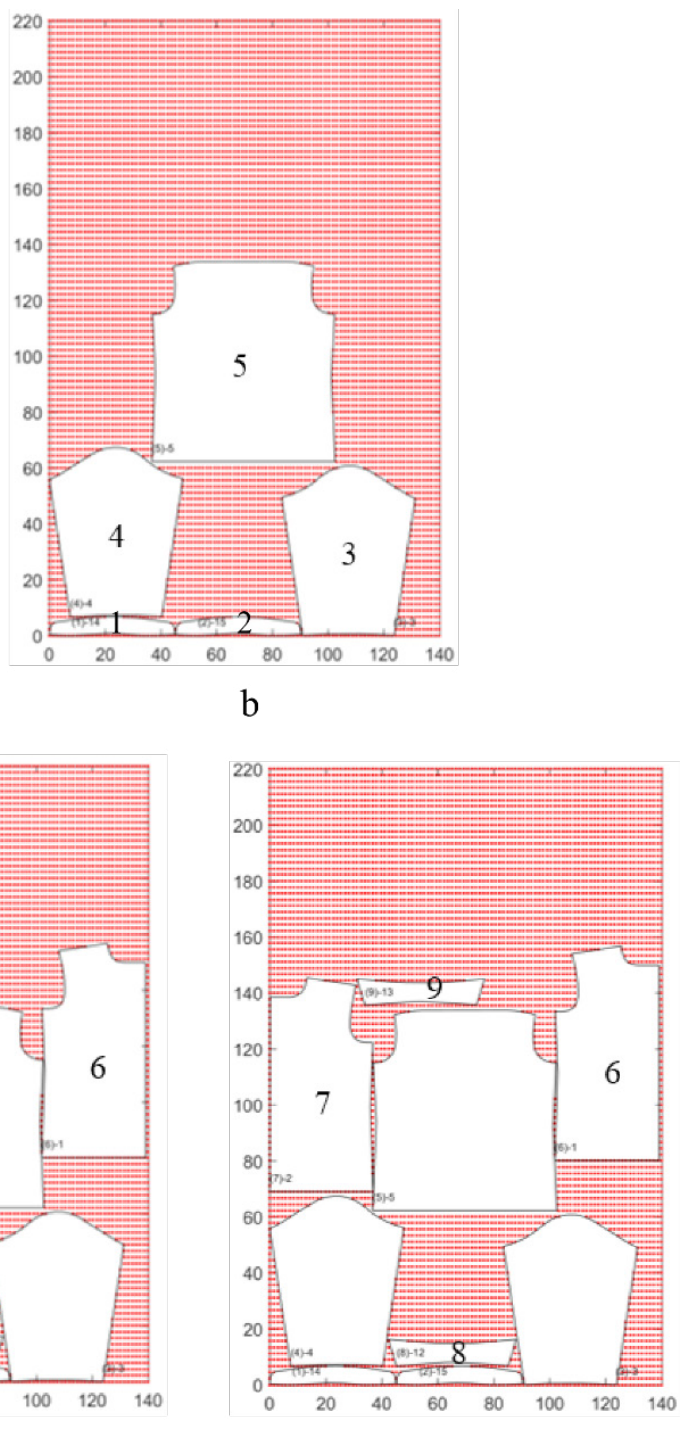

$\mathrm{e}$

Figure 2. An example of cutting part placement on grid points and in the waste space

BLP. Grid heuristic is used to place the cutting part on its initial feasible position. Since cutting part is an irregular polygon, after choosing the position based on grid, waste space may appear between the cutting part and previously placed cutting parts. Therefore, a BLP is applied on the cutting part, as defined in [32]. Starting from the current position in a layout, a cutting part is moved iteratively down and left, until a stable position is found.

It has been noticed that sometimes a cutting part could have been placed even lower and left in the marker, but that placement has not been obtained due to the specific shape of a cutting part, and its relation to the temporary layout. A cutting part may have been caught in some local optima.

Therefore, a heuristic called Grid-Shaking has been introduced in this paper in order for the cutting parts to escape local optima. Shaking heuristic uses a procedure to escape local optima that moves the cutting part away from the stable position in which it has been trapped after the BLP has found a stable position. It combines the BLP (Figure 3b, 3d) and BRP (bottom-right, Figure $3 \mathrm{c}$ ) procedure iteratively. A BRP procedure is performed on the cutting part as a sequence of horizontal right and down movements, until a stable position is found (Figure 3c). Grid heuristic is used to place cutting part on its initial feasible position (Figure 3a).

A flowchart of the Grid variants is shown in Figure 5.

\subsection{Placement order All equal first (AEF)}

In this research, the cutting parts are placed on a marker in the order determined by an individual in GA. While examining the datasets used in this research, it has been discovered that some of the cutting parts have their identical replicas within the same dataset (e.g., front and back cutting part of a shirt). For example, as defined in [26], the dataset ALBANO is composed of 24 cutting parts, while there are only eight different groups of cutting parts - four groups of 4 , and four groups of 2 identical replicas of the same cutting part.

Placement of groups of equal cutting parts named AEF (All Equal First) has been introduced. AEF placement order has been used since experts, based on their experience, tend to 


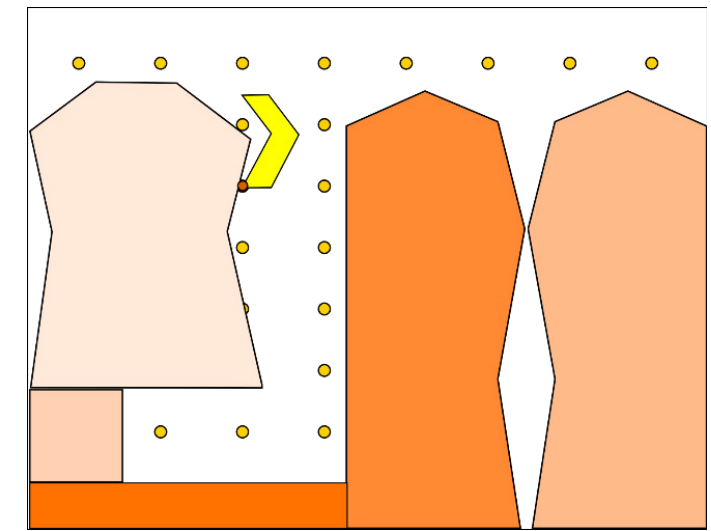

a)

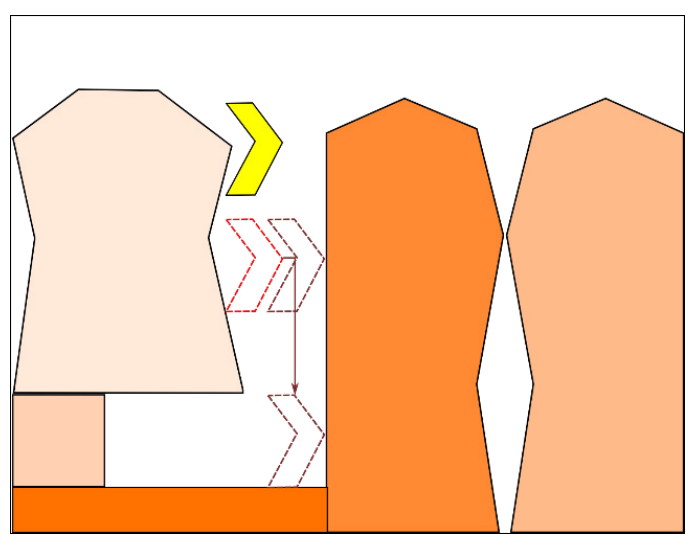

c)

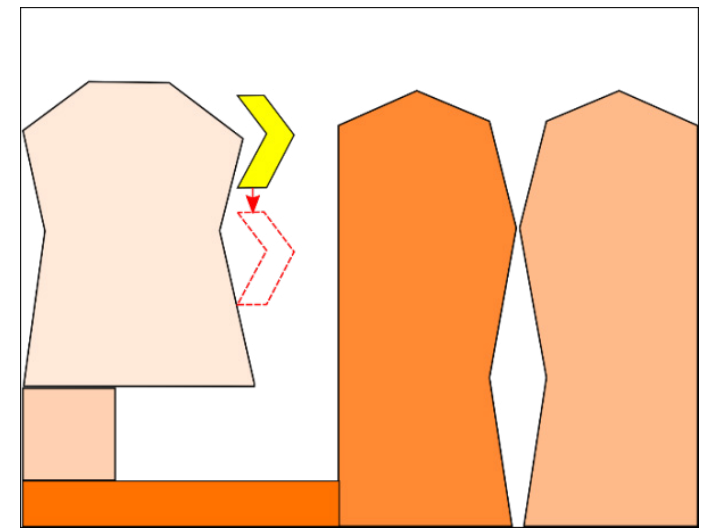

b)

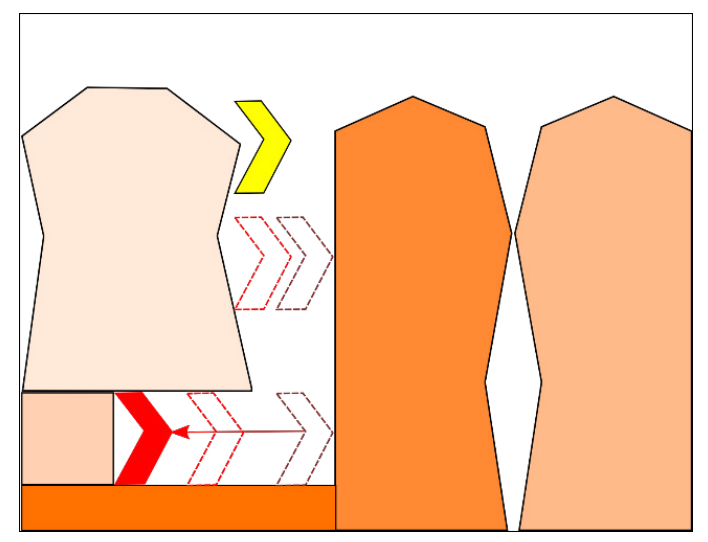

d)

Figure 3. Grid-Shaking

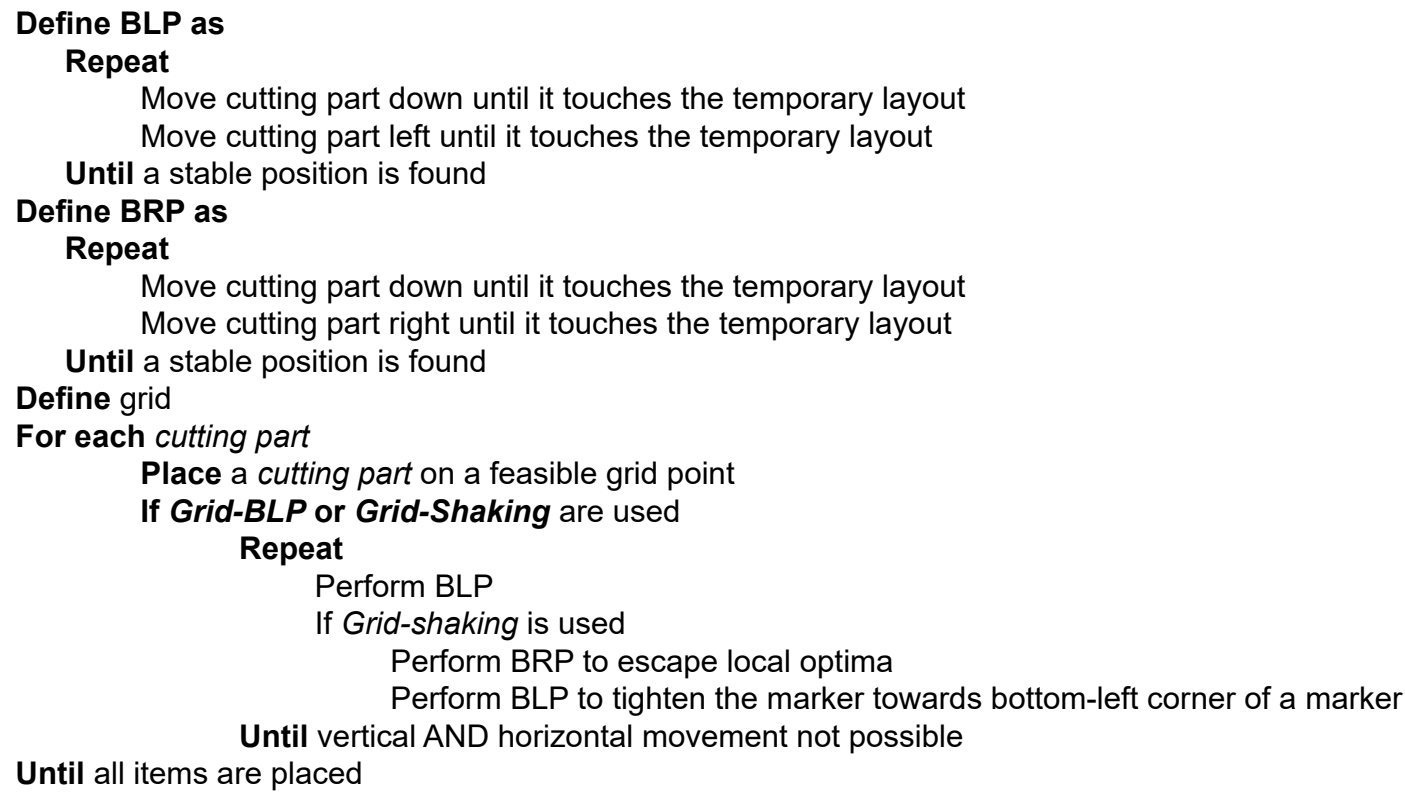

Move cutting part down until it touches the temporary layout

Move cutting part right until it touches the temporary layout

Until a stable position is found

Define grid

For each cutting part

Place a cutting part on a feasible grid point

If Grid-BLP or Grid-Shaking are used

Repeat

Perform BLP

If Grid-shaking is used

Perform BRP to escape local optima

Perform BLP to tighten the marker towards bottom-left corner of a marker

Until vertical AND horizontal movement not possible

Until all items are placed

Figure 4. Pseudocode of Grid variants

group equal cutting parts together, and place bigger cutting parts on the marker first [1].

The main benefit of AEF is reducing the search space. When placing each cutting part at a time, the search space would have been $n$ ! (i.e., the number of different permutations in a lexicographic order), where $n$ is the number of cutting parts in a dataset. If $n g r(n g r<n)$ is the number of groups of equal cutting parts in a dataset, the search space is reduced to ngr!.

AEF has been used for obtaining the placement order sequence as a part of an individual in genetic algorithm. 


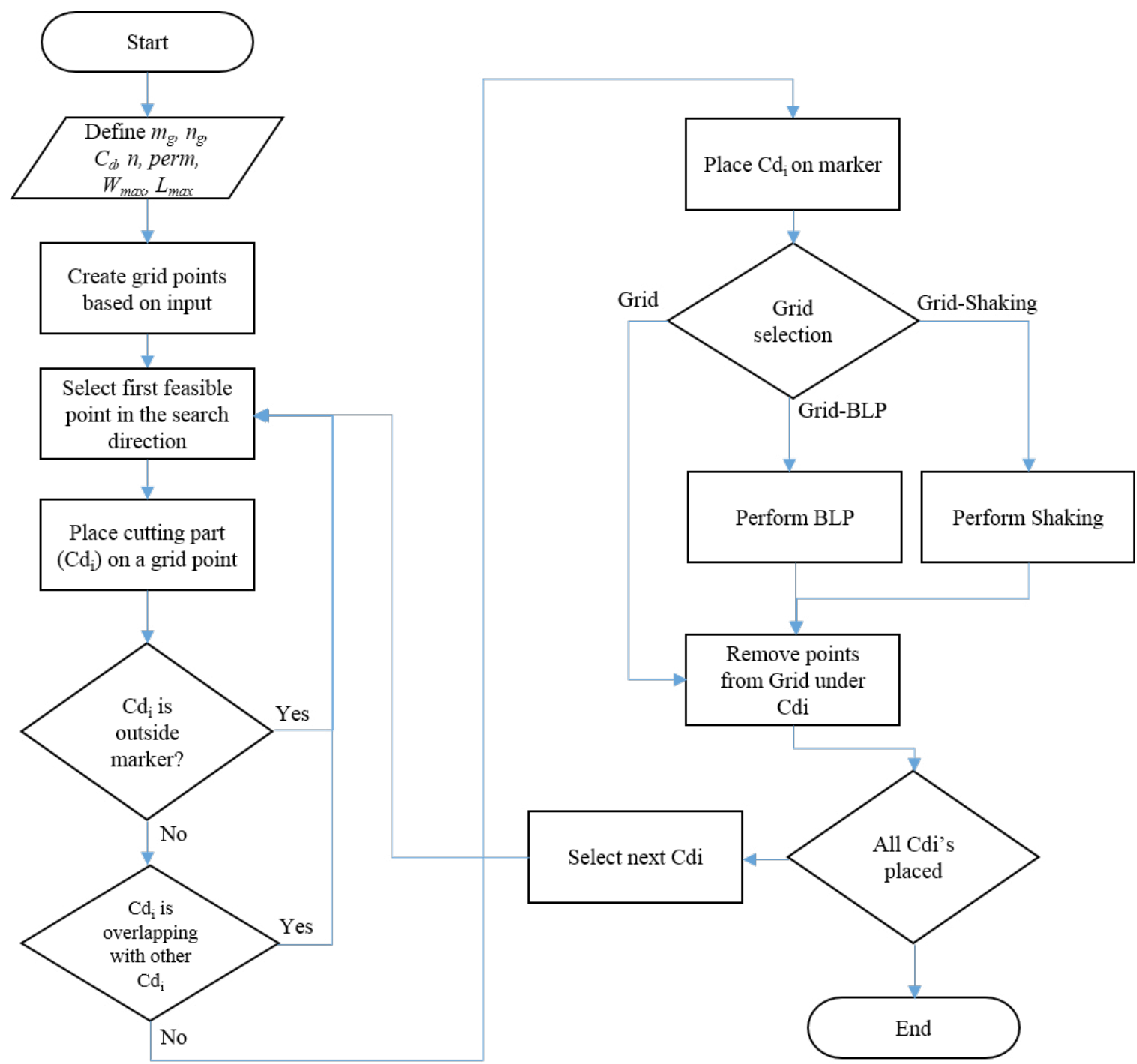

Figure 5. Block diagram of Grid, Grid-BLP and Grid-Shaking heuristic

\subsection{Genetic Algorithm}

Genetic algorithm (GA) is an evolutionary algorithm (EA) that mimics the principles of biological evolution [33]. Evolutionary algorithms look for the best solution in a search space. A search space is a set of all possible solutions to a problem. Evolutionary algorithms operate on a set of individuals called population. Individuals are potential solutions of a problem. One possible solution is represented by one individual in a population. In genetic algorithm, an individual is called a chromosome. Chromosomes are composed of genes.

The optimization process of EA is iterative. Individuals in a population are iteratively improved from generation to generation by applying the evolution principle of survival of the fittest. A population in each iteration is called a generation.

The fitness of individuals is evaluated in each generation by obtaining the value of the objective function for the specific optimization problem that is being solved.

Optimization process starts by randomly generating an initial population, followed by the selection stage in which individuals are chosen from a population for the mating process (crossover and mutation). Individuals are selected based on their fitness value. Individuals with higher fitness have greater likelihood of being selected for mating.

Crossover is a genetic operator that uses selected individuals to produce offspring, that is, new solutions to the problem. Selected individuals are considered to pass good properties to their offspring and improve the population quality. The process is analogous to reproduction and biological crossover. Offspring undergoes the process of mutation. The purpose of mutation is for solutions to escape local extrema.

The iterative process ends when a termination criterion is met. Possible termination criteria include: maximal number of iterations, execution time, number of fitness function evaluations etc [34].

\section{Novel individual representation for GA}

In this research, GA is hybridized with Grid methods to obtain high quality results. In order to implement GA to obtain markers with Grid heuristics and AEF placement order, a novel representation of an individual in GA had to be developed. 
An example of a novel individual representation is depicted in Figure 6.

Each individual is composed of three parts:

(1) Permutation that determines the placement order of cutting parts

(2) Rotation of cutting parts

(3) Placement heuristic (hyper-heuristic)

The first part of an individual is permutation representation of a placement order. The permutation defines the order in which cutting parts are placed on a marker. For example, in Figure 6, the fifth cutting part from a dataset will be placed on the marker first, followed by the third cutting part and so on.

In order to implement the AEF placement order, a permutation part of an individual is represented as an integer (Figure 7), which is later decoded to permutation.

The reason the individual has the following structure was imposed for two reasons. The first one lies in the shortcomings of the GEATbx toolbox, which is a crucial part of our application, since it guides the optimization process using the genetic algorithm [35]. GEATbx may represent individuals as: integer numbers, real numbers and permutation, but it does not provide the possibility for multiple data type representation at the same time (e.g. a part of an individual is integer, while the other part is permutation) [36]. Since there is a natural mapping between integers when presented in a factoradic form and permutations, a factoradic transformation was used to decode the integer value into the permutation sequence. That means the genetic algorithm in GEATbx takes individuals in the form of integers, while the fitness function reconstructs integers into permutation sequence.

The second reason for using AEF is in MATLAB's precision [37]. When calculating permutation of $n$ for double-precision inputs, the result is exact when $n$ is less than or equal to 21 .
Larger values of $n$ produce a result that has the correct order of magnitude and is accurate for the first 15 digits. This is because double-precision numbers are only accurate up to 15 digits. When calculating permutation of $n$ for single-precision inputs, the result is exact when $n$ is less than or equal to 13 . Larger values of $n$ produce a result that has the correct order of magnitude and is accurate for the first 8 digits. This is because single-precision numbers are only accurate up to 8 digits.

Mapping between integers and permutations is done using the factorial number system used by, [38] which is also known as factoradic in combinatorics. It is a mixed radix numeral system used for numbering permutations. If a number less than $n$ ! is converted to factorial representation, a sequence of $n$ digits is obtained that can be converted to a permutation of $n$, either by using them as Lehmer code or as inversion table representation as in [39]. An algorithm for such a mapping is presented in [38].

A decoding procedure had to be developed to transform the placement sequence of a group of equal elements into placement order of individual elements (Figure 8). An integer part of the individual is transformed to factoradic form perm, and then into a permutation sequence seq representing groups of cutting parts, which are supposed to be placed on a marker based on the lexicographic order from this permutation. Perm is composed of $n c p$ elements indicating groups of elements and is subjected to a decoding process to obtain the placement order of $n$ elements. An example of decoding a permutation perm that defines the order of groups into permutation seq and the order of cutting parts as well is shown in Figure 9.

The second part of an individual determines the rotation of a cutting part. Each gene can take the integer value $n$ from a set $\{0,2\}$. Rotation angle is $n \times 90^{\circ}$. The cutting parts are not rotated in a random direction. Each rotation gene is connected to its correspondent order gene. If $c p i$ is the $i$-th cutting part in a dataset, and $n$ is the number of cutting parts in a dataset,

\begin{tabular}{|l|c|c|c|c|c|c|c|c|c|c|c|c|c|c|c|c|}
\hline 5 & 3 & 7 & 2 & 1 & 4 & 6 & 8 & 0 & 0 & 2 & 0 & 2 & 2 & 0 & 2 & 3 \\
\hline
\end{tabular}

Figure 6. Example of an individual representation for dataset with eight cutting parts

\begin{tabular}{|l|l|l|l|l|l|l|l|l|l|}
3845 & 0 & 0 & 2 & 0 & 2 & 2 & 0 & 2 & 3 \\
\hline
\end{tabular}

Figure 7. Individual representation using Radix

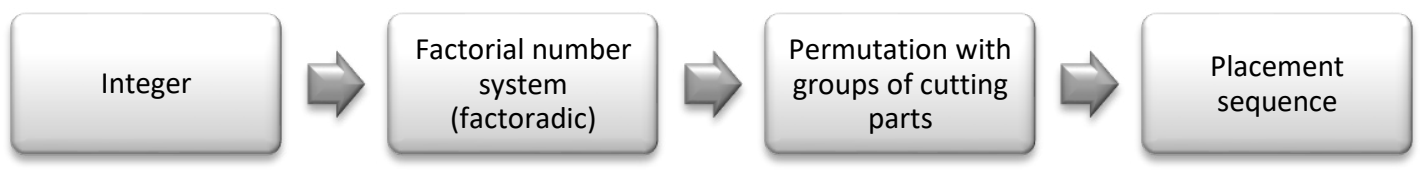

Figure 8. Mapping between integers and permutation

\begin{tabular}{|c|c|c|c|c|c|c|c|}
\hline Perm & 5 & 4 & 1 & 6 & 3 & 2 \\
\hline seq & $\begin{array}{c}13,14,15, \\
16\end{array}$ & $\begin{array}{c}9,10,11, \\
12\end{array}$ & 1,2 & $\begin{array}{c}17,18,19, \\
20\end{array}$ & $5,6,7,8$ & 3,4 \\
\hline
\end{tabular}

Figure 9. Decoder example for ALBANO dataset for seq $=\left[\begin{array}{lllll}13 & 14 & 16 & \ldots\end{array} 2122\right]$ 
the corresponding rotation gene has index $n+i$ within the chromosome.

The third part of the individual is the hyper-heuristic parameter that determines the heuristic that will place the cutting parts on the marker. The value is an integer number from a set $\{1,2,3\}$, where 1 stands for Grid heuristics, 2 for Grid-BLP heuristic and 3 for Grid-Shaking heuristic.

\subsection{Hyper-heuristic approach}

Besides Grid, Grid-BLP and Grid-Shaking heuristics, a hyperheuristic approach is developed, named Grid-all, which chooses and applies one of the three different heuristics for cutting parts' placement.

The Grid-all hyper-heuristic is a flexible method that uses a novel individual representation of GA. Grid-all decides which heuristic will be used to obtain a marker. The choice is performed as a parameter of GA. Therefore, each individual in one generation of GA may use different heuristic for cutting parts' placement. This increases the search space providing a wider range of possible solutions, while enabling convergence inherent to GA. The chosen heuristic may be either Grid, GridBLP or Grid-Shaking. The pseudocode for the hyper-heuristic Grid-all is shown in Figure 10.
According to the hyper-heuristic taxonomy in [21], the hyperheuristic implemented in this research belongs to the group of hyper-heuristics for heuristic selection since it chooses between three lower-level heuristics. Also, the implemented approach is a perturbative method since heuristics of the lower level seek to improve the potential solution through the process of automatic selection and application of heuristics. Since hyper-heuristic is implemented within the evolutionary algorithm, learning is left to the operators of the evolutionary algorithm, so it can be said that the hyper-heuristic used in this research has no learning ability. However, as the results show, the evolutionary algorithm chooses the most suitable heuristics for obtaining a marker.

\subsection{Data sets}

In this research experiments to investigate marker making are conducted on datasets: ALBANO, DAGLI, MAO and MARQUES, taken from garment industry, that can be obtained from [26]. These datasets are polygonal approximations of real cutting parts. Approximations have been performed to lower computational complexity. A new dataset called MAN SHIRT has been created for this research, which is composed of real cutting parts of a man shirt in one size. The information about datasets is shown in Table 1.

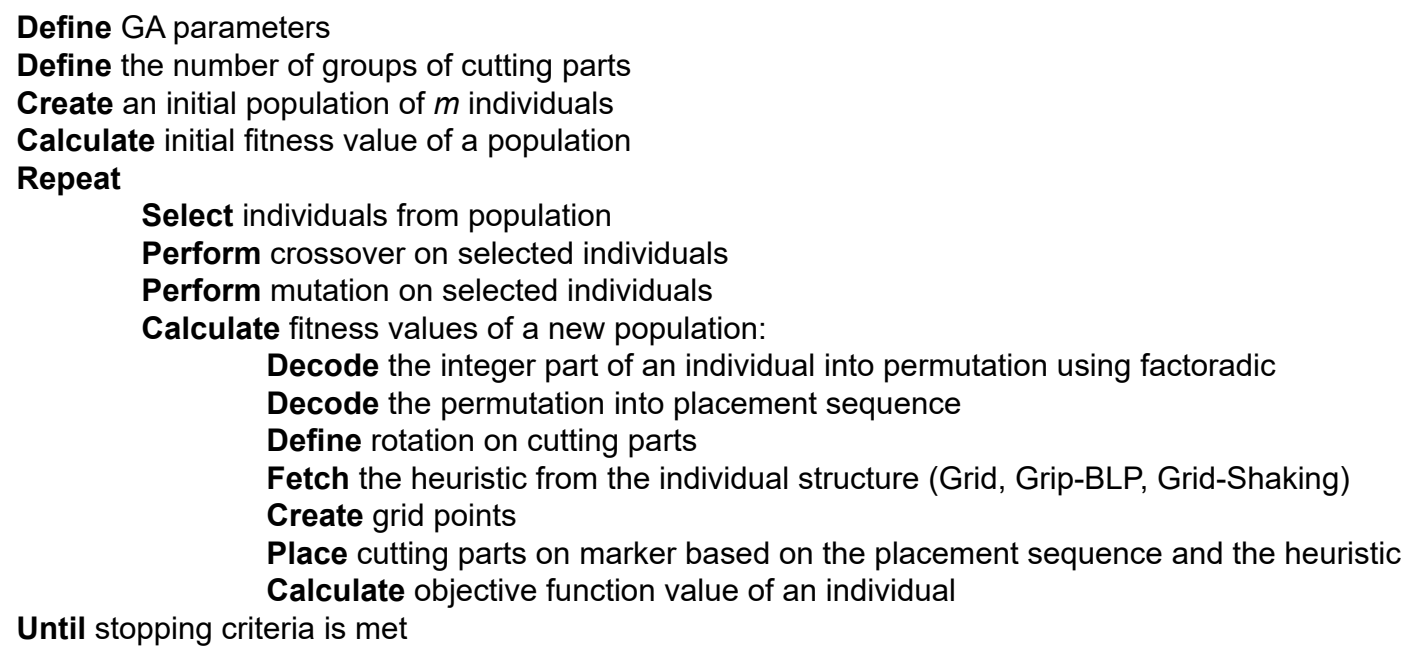

Figure 10. Hyper-heuristic Grid-all

Table 1. Datasets features

\begin{tabular}{|c|c|c|c|c|c|c|}
\hline Dataset & $\begin{array}{l}\text { Number of } \\
\text { different } \\
\text { pieces }\end{array}$ & $\begin{array}{l}\text { Number } \\
\text { of cutting } \\
\text { parts }\end{array}$ & Width & Rotations & Shapes & Source \\
\hline ALBANO & 8 & 24 & 4900 & \multirow{5}{*}{0,180} & \multirow{5}{*}{$\begin{array}{l}\text { Polygons, non-polygonal } \\
\text { pieces with arcs }\end{array}$} & \multirow{4}{*}{$\begin{array}{c}\text { Scanned from sample } \\
\text { layout in paper; } \\
\text { approximated by polygons }\end{array}$} \\
\hline DAGLI & 10 & 30 & 60 & & & \\
\hline MAO & 9 & 20 & 2550 & & & \\
\hline MARQUES & 8 & 24 & 104 & & & \\
\hline MAN SHIRT & 10 & 17 & 140 & & & $\begin{array}{l}\text { Real cutting part made in } \\
\text { Lectra }\end{array}$ \\
\hline
\end{tabular}




\subsection{Experimental environment and parameters}

Applications have been developed in MATLAB. GEATbx (Genetic and Evolutionary Algorithms toolbox) has been used for genetic algorithm [35].

\section{GA parameters}

Parameters used in GEATbx are described in Table 2. Each generation is composed of 25 individuals (Nind) in 50 generations (Ngen). Individual with the highest fitness value found upon this termination criterion is considered to be the best solution to the problem. A total of ten experiments (Nexp) for each dataset has been run.

During the preliminary research, experiments have been conducted with 100 generations of GA. It has been concluded that a significant change in the fitness function value does not occur after $50^{\text {th }}$ generation (Figure 11) or the desired progress is not significant. Therefore, a value of 50 generations has been chosen resulting in faster performance.

Initial population is created randomly. In the selection process, a stochastic universal sampling (SUS) is used as described in [40], alongside discrete recombination [41] and integer mutation [36].

Stochastic universal sampling is used for selection with pressure factor of 1.6 and generation gap of 0.96 . The value of selective pressure determines the fitness assignment and is used by the ranking algorithm. Generation gap (GP) defines the portion of population that will be reproduced in every generation. The parameter determines the number of individuals to be produced with respect to the number of individuals in the population [35]. If the GP value is 1, all parent individuals are substituted with their offspring. If the GP value is less than 1, elitism is included since less offspring than parents is created; therefore, parents with best fitness survive and are a part of the next generation. In this research, 24 new offspring will be produced $(0.96 \times 25=$ 24 ) in each generation and one individual is always kept as the best individual (elitism).

Discrete recombination performs the exchange of genes (variables) between individuals based on a randomly generated mask defining which parent contributes its variable to their offspring, with the same probability of 0.5 .

Table 2. GEATbx parameters for Grid heuristics

\begin{tabular}{|c|c|c|}
\hline \multicolumn{2}{|c|}{ Parameter } & Value \\
\hline \multicolumn{2}{|c|}{$\mathrm{N}_{\text {ind }}$} & 25 \\
\hline \multicolumn{2}{|c|}{$\mathrm{N}_{\text {gen }}$} & 50 \\
\hline \multicolumn{2}{|c|}{$N_{\text {exp }}$} & 10 \\
\hline \multirow{3}{*}{ Selection } & Name & SUS \\
\hline & Selective Pressure & 1.6 \\
\hline & Generation Gap & 0.96 \\
\hline Recombination & Name & $\begin{array}{c}\text { Discrete } \\
\text { recombination }\end{array}$ \\
\hline Mutation & Name & Integer mutation \\
\hline Termination & Name & Maximum number of generations \\
\hline
\end{tabular}

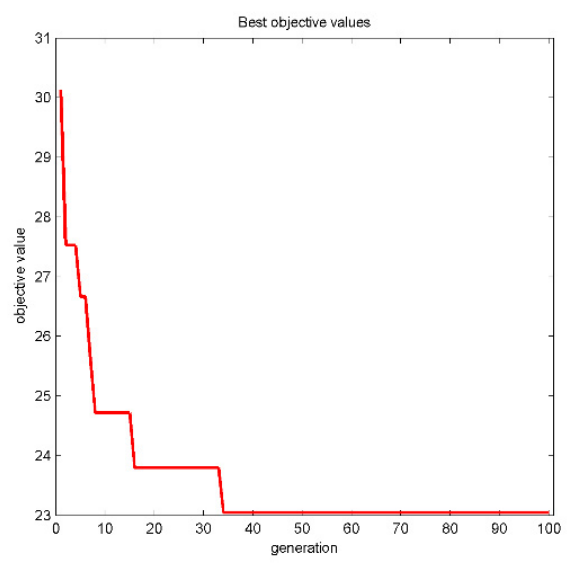

a)

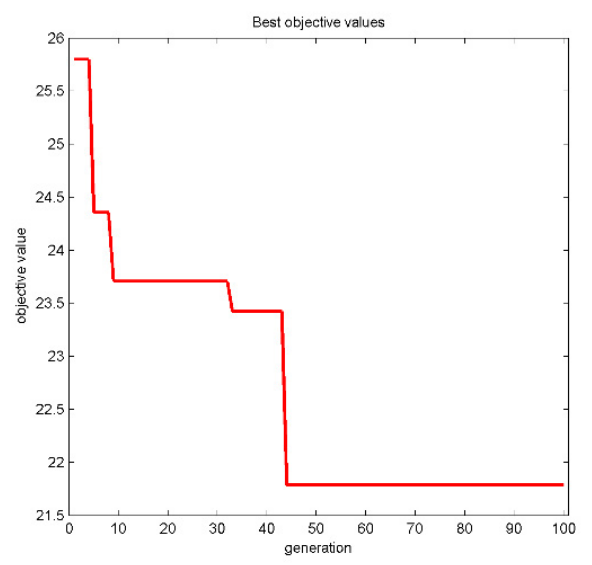

b)

Figure 11. Fitness function in experiments with 100 generation of GA 


\begin{tabular}{|c|c|c|c|c|c|c|c|c|}
\hline Parent 1 & 15 & 27 & 35 & 45 & 11 & 7 & 8 & 87 \\
\hline Parent 2 & 13 & 54 & 48 & 12 & 74 & 1 & 85 & 64 \\
\hline Mask 1 & 1 & 1 & 2 & 1 & 2 & 2 & 1 & 2 \\
\hline Mask 2 & 2 & 2 & 1 & 2 & 1 & 1 & 2 & 1 \\
\hline Offspring 1 & 15 & 27 & 48 & 45 & 74 & 1 & 8 & 64 \\
\hline Offspring 2 & 13 & 54 & 35 & 12 & 11 & 7 & 85 & 87 \\
\hline
\end{tabular}

Figure 12. Example of discrete recombination

Integer mutation is performed by adding randomly generated values to some genes with a low probability. The probability of mutation (mutation rate) and the size of change for each mutated gene (mutation step) must be defined. The mutation probability is inversely proportional to the number of genes. With an increase in the number of genes, the probability of mutation decreases. The probability of mutation is independent of the size of the population.

The number of grid points in this research is set to $100 \times 100$ based on the results of preliminary experiments.

Efficiency of a marker Em is calculated as in (6):

$$
E_{m}=A_{p} / A_{p} \cdot 100[\%]
$$

where Acp is the area of cutting parts, and Alp is the total area of the marker after the heuristic placement.

\section{Results and discussion}

Four novel methods for automatic marker making were introduced in this research and compared for its efficiency. The most basic one is the Grid heuristic that places the cutting part on the first available grid point that satisfies the conditions. Compaction methods: Grid-BLP and Grid-Shaking improve Grid heuristic's efficiency by reducing the waste between cutting parts. Therefore, the following hypothesis have been posed:

(1) The Grid-BLP and Grid-Shaking will obtain higher marker efficiency than the Grid heuristic.

(2) The Grid-Shaking heuristic will obtain the best results for all datasets since it can escape the local optima.

A research has also been conducted to determine the efficiency of hyper-heuristic placement and to determine which combination of a grid heuristic creates the best efficiency marker with genetic algorithm and AEF placement.

In order to do so, experiments have been conducted with Grid, Grid-BLP and Grid-Shaking heuristics individually to determine which placement method obtains the most efficient markers. This is then compared with the hyper-heuristic placement Gridall results to determine how often the most efficient placement method has been chosen by the hyper-heuristic, what is the quality of obtained solutions, and to confirm:
(3) Grid-all will produce markers with highest efficiency.

(4) Grid-all will choose the most efficient heuristic in most cases.

The created algorithm has been used to create mixed multisize markers since the datasets do not have to be composed of cutting parts of equal size. The efficiency of a marker is commonly best cutting parts of different sizes can interlock [3].

For each dataset and method, 10 experiments have been conducted and several parameters have been recorded and presented in Table 3 and Table 4: the worst (MIN) and the best (MAX) obtained marker efficiency, the arithmetic mean (AVG) and median (MED) of the 10 measurements (the results of marker efficiencies are shown in percentage), the range between the minimum and the maximum solution (RAN), standard deviation (STD), variance (VAR) and the standard error (ERR), and the number of times Grid-all hyper-heuristic has chosen the Grid, Grid-BLP or Grid-Shaking heuristic (EFF). Parameter median has been recorded since the outliers may be obtained by using genetic algorithms that influence the statistic quality, but it can be seen that there is no significant difference between arithmetic mean and median value.

The Tables 3 and 4 show the results on benchmark data sets described in section 2.5, obtained by Grid heuristic (G1), GridBLP heuristic (G2), Grid-Shaking heuristic (G3) and the hyperheuristic Grid-all (G4).

First, the efficiency of markers obtained by Grid, Grid-BLP and Grid-Shaking heuristic is analyzed. The efficiency of a marker obtained by the Grid-BLP and Grid-Shaking heuristic always improved the solution obtained by the Grid heuristic. Grid heuristic obtains the worst results for all datasets.

Figure 13 shows the box-plot of the results for grid heuristics (G1-G4) applied on all datasets (D1-D5). The best median values are obtained by Grid-Shaking (except for ALBANO, but for this data set the median is approximately equal to the median of Grid-BLP heuristic), which proved hypothesis (1) and (2) to be valid and proves the Grid-BLP and Grid-Shaking have the ability to escape the possible local optima.

The results of the hyper-heuristic approach are shown in Tables 3 and 4 in column G4. Hyper-heuristic is able to select and apply either Grid, Grid-BLP or Grid-Shaking heuristic for each individual in a generation of GA.

In Table 5, the percentage of low-level heuristic choices obtained by hyper-heuristic Grid-all are presented. In most cases, Grid-Shaking was selected and obtained the best result. In the remaining cases, a Grid-BLP heuristic had obtained better results.

Although hyper-heuristic didn't always use Grid-Shaking, which is due to the fact of increasing the search space, that is, three different heuristics can be chosen instead of only one as in individual heuristic experiments, it has to be mentioned that the overall best results (column MAX shown in Tables 3 
Table 3. Obtained results for datasets ALBANO, DAGLI AND MAO

\begin{tabular}{|c|c|c|c|c|c|c|c|c|c|c|c|c|}
\hline & \multicolumn{4}{|c|}{ ALBANO } & \multicolumn{4}{|c|}{ DAGLI } & \multicolumn{4}{|c|}{ MAO } \\
\hline & G1 & G2 & G3 & G4 & G1 & G2 & G3 & G4 & G1 & G2 & G3 & G4 \\
\hline MIN & 77.85 & 79.68 & 77.66 & 81.53 & 72.88 & 74.56 & 76.62 & 76.58 & 70.06 & 71.14 & 72.14 & 71.59 \\
\hline MED & 78.52 & 81.28 & 81.25 & 82.65 & 75.05 & 75.89 & 78.16 & 78.53 & 71.73 & 74.54 & 74.71 & 74.87 \\
\hline AVG & 78.50 & 81.41 & 81.18 & 82.60 & 74.96 & 75.93 & 77.80 & 78.54 & 72.17 & 74.19 & 75.02 & 74.58 \\
\hline MAX & 79.60 & 83.16 & 83.24 & 84.50 & 77.37 & 77.85 & 79.04 & 80.13 & 74.70 & 76.28 & 79.54 & 77.43 \\
\hline RAN & 1.75 & 3.48 & 5.58 & 2.97 & 4.49 & 3.28 & 2.42 & 3.55 & 4.64 & 5.14 & 7.40 & 5.84 \\
\hline STD & 0.43 & 1.03 & 1.52 & 0.82 & 1.26 & 0.90 & 0.84 & 1.10 & 1.78 & 1.77 & 2.22 & 2.10 \\
\hline VAR & 0.18 & 1.06 & 2.30 & 0.67 & 1.60 & 0.81 & 0.70 & 1.21 & 3.17 & 3.13 & 4.93 & 4.42 \\
\hline ERR & 0.14 & 0.33 & 0.48 & 0.26 & 0.40 & 0.29 & 0.26 & 0.35 & 0.56 & 0.56 & 0.70 & 0.66 \\
\hline EFF & 0 & 0 & 10 & - & 0 & 1 & 9 & - & 0 & 2 & 8 & - \\
\hline
\end{tabular}

Table 4. Obtained results for datasets MARQUES and MAN SHIRT

\begin{tabular}{|c|c|c|c|c|c|c|c|c|}
\hline & \multicolumn{9}{|c|}{ MARQUES } & \multicolumn{3}{c|}{ MAN SHIRT } \\
\hline & G1 & G2 & G3 & G4 & G1 & G2 & G3 & G4 \\
\hline MIN & 75.83 & 79.50 & 77.78 & 79.50 & 75.81 & 78.80 & 82.89 & 79.51 \\
\hline MED & 79.21 & 82.04 & 83.34 & 82.38 & 77.32 & 81.28 & 84.96 & 84.05 \\
\hline AVG & 79.14 & 82.23 & 82.29 & 81.92 & 77.04 & 82.00 & 84.63 & 83.38 \\
\hline MAX & 83.45 & 84.36 & 84.35 & 84.67 & 77.93 & 85.12 & 86.02 & 85.60 \\
\hline RAN & 7.62 & 4.86 & 6.56 & 5.18 & 2.11 & 6.32 & 3.13 & 6.09 \\
\hline STD & 2.30 & 1.34 & 2.14 & 1.81 & 0.68 & 2.13 & 0.99 & 1.97 \\
\hline VAR & 5.28 & 1.81 & 4.57 & 3.29 & 0.47 & 4.53 & 0.99 & 3.87 \\
\hline ERR & 0.73 & 0.43 & 0.68 & 0.57 & 0.22 & 0.67 & 0.31 & 0.62 \\
\hline EFF & 0 & 2 & 8 & - & 0 & 1 & 9 & - \\
\hline
\end{tabular}

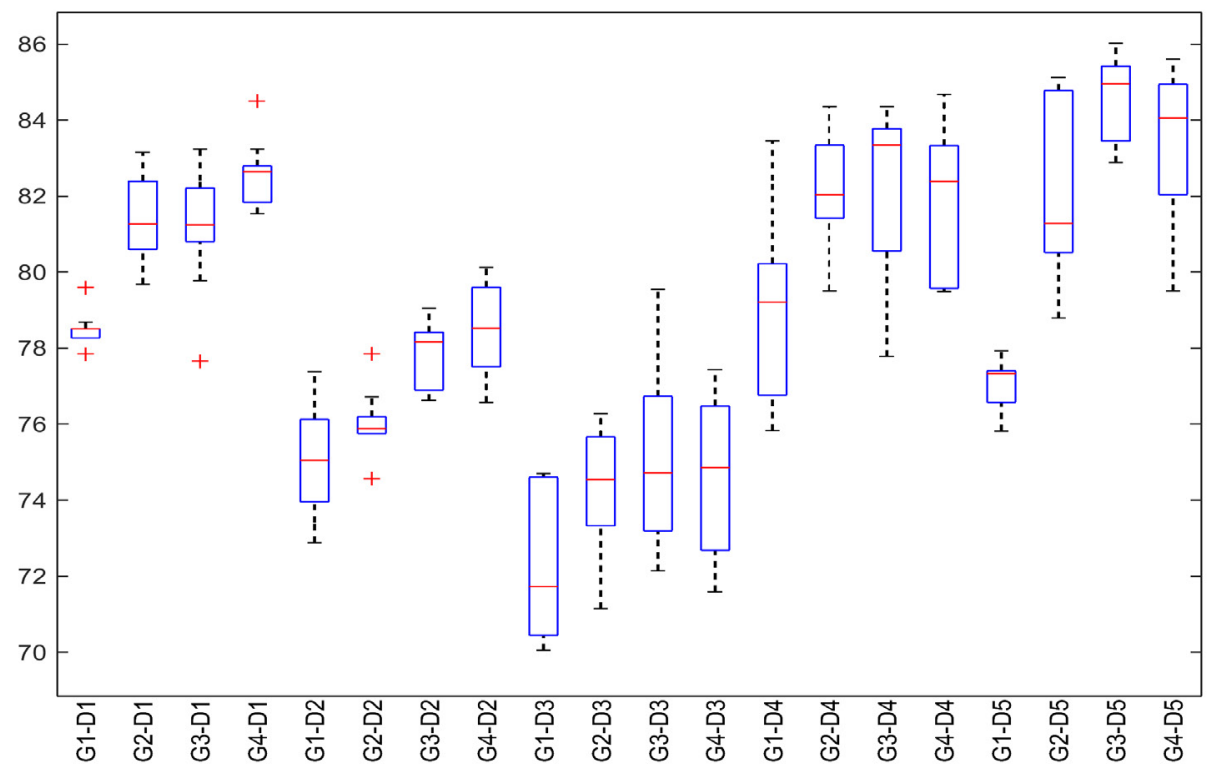

Figure 13. Box-plot of results for of a Grid heuristic (G1), Grid-BLP heuristic (G2), Grid-Shaking heuristic (G3) and the hyper-heuristic (G4) on datasets ALBANO (D1), DAGLI (D2), MAO (D3), MARQUES (D4) and MAN SHIRT (D5) 
and 4 for Grid-Shaking) have always been obtained when Grid-Shaking was chosen by the hyper-heuristic. Therefore, it may be concluded that hypothesis (3) is partially valid and hypothesis (4) is valid.

Since in this application we are interested in the best obtained result, in Figure 14, a comparison of the overall best obtained results for all datasets is shown.

Grid-Shaking obtains similar or better results than the Grid and Grid-BLP heuristic. Results indicate that the results obtained by Grid-all are usually better, or slightly inferior to the GridShaking heuristic, which may come from the fact Grid-all deals with a larger search space.

The overall best results obtained in this research have been noted in Table 6 and compared with the published results from the literature and commercial programs.

In [32], a BLF (bottom-left-fill) heuristic was used and hybridized with evolutionary algorithms. The features of the algorithm are similar to the ones developed in this research. Using BLF, the waste space between polygons can be filled, and the rotation of cutting parts is allowed. Our proposed methodology outperforms the ones in [32] on all examined datasets. In [15], a modification of BLF method that is able to nest polygons with arcs and holes in a rectangular container is introduced, without discussing the possibility of extending the algorithm to a
Table 5. Count of uses of low-level heuristics by hyper-heuristic Grid-all

\begin{tabular}{|c|c|c|c|}
\hline \multirow{2}{*}{ Dataset } & \multicolumn{3}{|c|}{ Heuristic } \\
\cline { 2 - 4 } & Grid & Grid-BLP & Grid-SH \\
\hline ALBANO & 0 & 0 & 10 \\
\hline DAGLI & 0 & 1 & 9 \\
\hline MAO & 0 & 2 & 8 \\
\hline MARQUES & 0 & 2 & 8 \\
\hline MAN SHIRT & 0 & 1 & 9 \\
\hline Average & 0 & 0.12 & 0.88 \\
\hline
\end{tabular}

problem with irregular containers. In [42], a deterministic beam search algorithm is presented, based on the revised TOPOS algorithm. A revised version of the no-fit polygon is used for overlap detection. The solution is viewed as an ordered list and decoded using constructive heuristic. Placement of cutting parts to irregular containers has not been discussed in the paper. The strength of the algorithm in [43] is the fast neighborhood search of positions obtained by translating polygons horizontally and vertically in order to obtain a position without overlapping. The neighborhood search is performed in polynomial time. A metaheuristic method Guided local search has been used to escape the local optima. Rotations of polygons in datasets MARQUES and MAO have been performed as increments of a $90^{\circ}$ angle, which may have benefited some of the results, but it cannot be

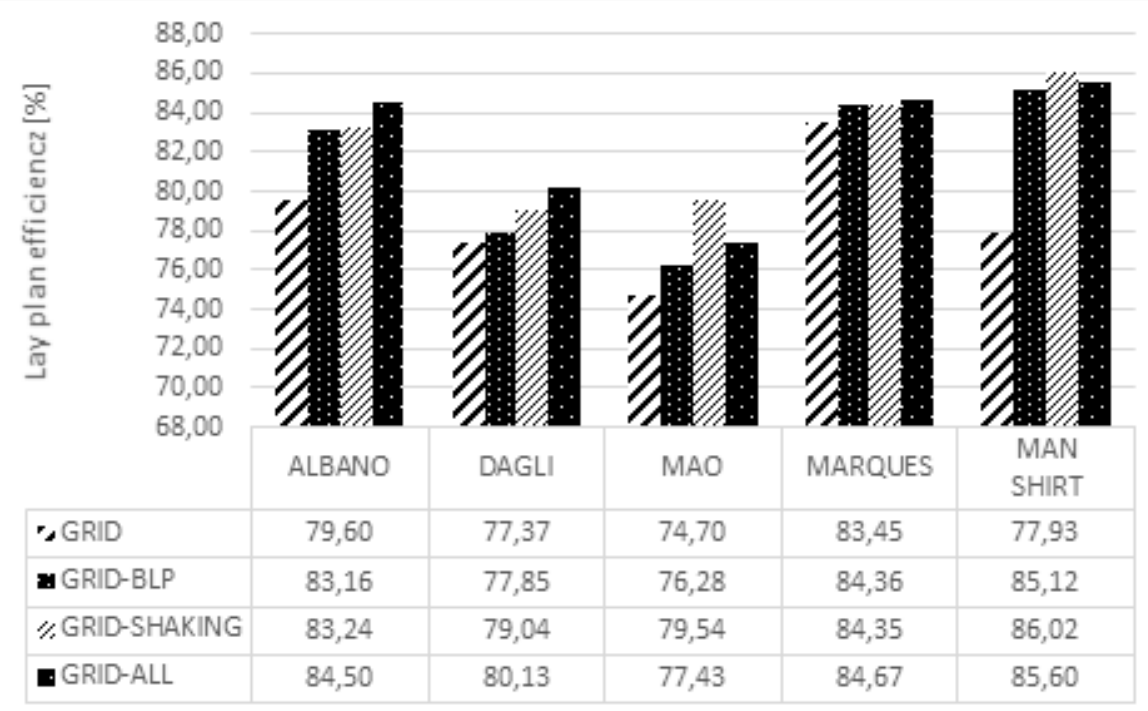

Figure 14. Comparison of the best obtained results of all methods on all test datasets

Table 6. Comparison with results in the literature and commercial programs

\begin{tabular}{|c|c|c|c|c|c|c|c|c|}
\hline $\begin{array}{c}\text { Authorl } \\
\text { Dataset }\end{array}$ & $\begin{array}{c}\text { Hopper } \\
\text { [32] }\end{array}$ & $\begin{array}{c}\text { Burke } \\
\text { [15] }\end{array}$ & $\begin{array}{c}\text { Egeblad } \\
\text { [42] }\end{array}$ & $\begin{array}{c}\text { Bennel } \\
\text { [43] }\end{array}$ & $\begin{array}{c}\text { Shalaby } \\
\text { [18] }\end{array}$ & $\begin{array}{c}\text { Optitex } \\
\text { [44] }\end{array}$ & $\begin{array}{c}\text { NestLib } \\
\text { [45] }\end{array}$ & $\begin{array}{c}\text { Grid } \\
\text { heuristics }\end{array}$ \\
\hline ALBANO & 84.09 & 84.60 & 87.88 & 87.88 & 83.36 & 86.37 & 73.66 & 84.50 \\
\hline DAGLI & 77.10 & 83.70 & 87.05 & 87.99 & 83.97 & 82.20 & 76.61 & 80.13 \\
\hline MAO & 68.65 & 79.50 & 85.15 & 84.07 & 78.40 & 79.42 & 75.36 & 79.54 \\
\hline MARQUES & 82.73 & 86.47 & 89.82 & 88.92 & 86.47 & 85.89 & 78.17 & 84.67 \\
\hline MANSHIRT & - & & - & - & - & - & - & 86.02 \\
\hline
\end{tabular}


performed when marker is created from textile materials with oriented structure. The SwarmNest algorithm in [18] uses the particle swarm optimization algorithm (PSO), a pixel method to obtain the initial layout, and direct trigonometry method for the refinement placement. The implementation focuses on hole-free polygons, whereas in our implementation, polygons may have holes, and approximation does not have to be used. Also, the placement of polygons in irregular containers is not discussed in the paper, and rotations of polygons for a $90^{\circ}$ angle increment are allowed. Experiments were performed on two commercial programs: Optitex [44] and NestLib [45]. Optitex is a program used to nest cutting parts in the garment

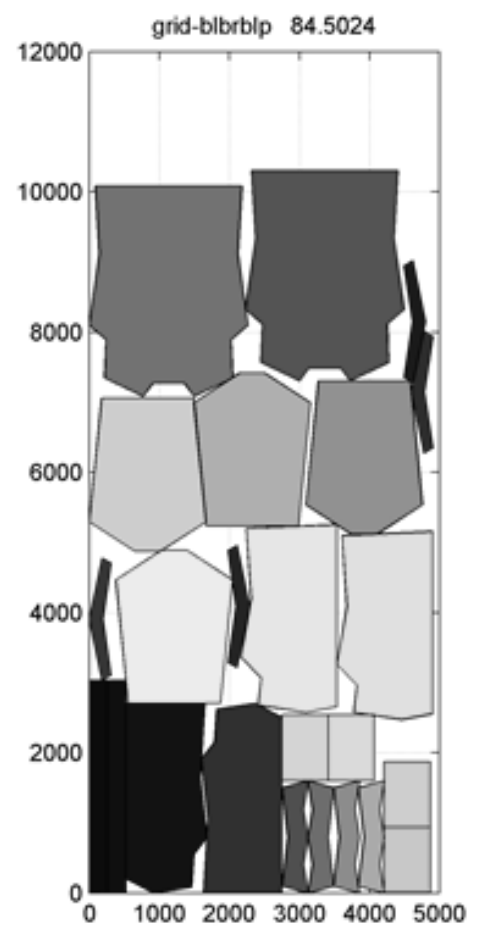

Figure 15. The overall best result for ALBANO

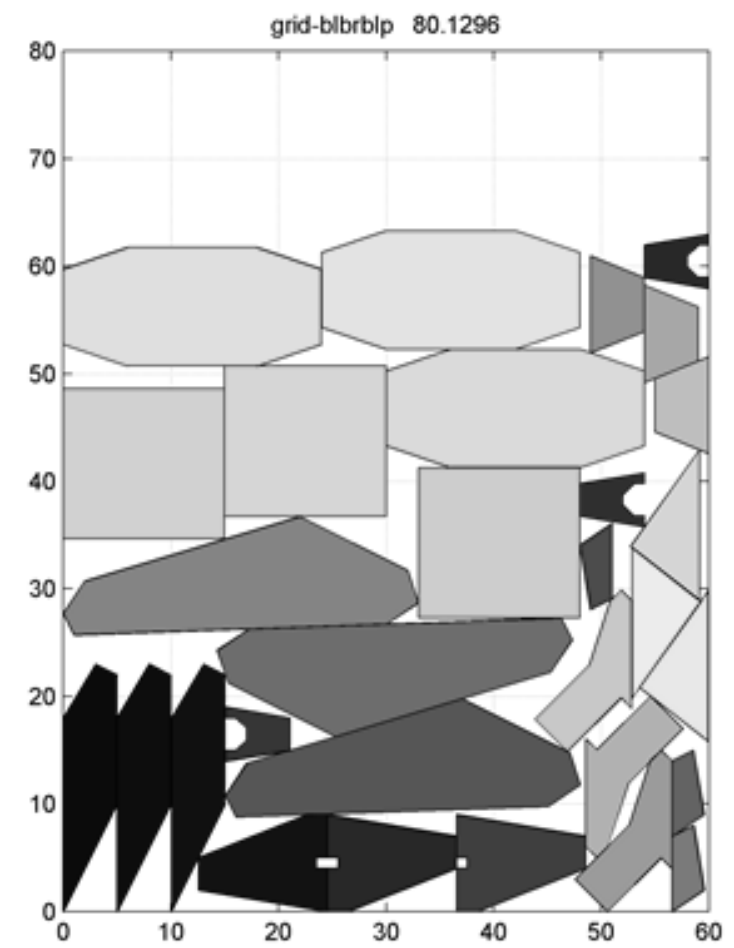

Figure 16. The overall best result for DAGLI industry. NestLib is a program used for nesting and optimizing material. Grid heuristics obtain better or competitive results as Optitex, and better results than the NestLib for all datasets.

In Figures 15 to 19, the overall best results are presented. The number above the marker represents the efficiency in percentage. If a name of a heuristic is mentioned before efficiency value, that marker has been obtained using hyperheuristic that applied the mentioned heuristic to obtain it.

\section{Future work and potential applications}

The created algorithms in this research have only been applied to marker making, but they have been designed with a goal of being adaptable and independent of the marker's and cutting parts' shape.

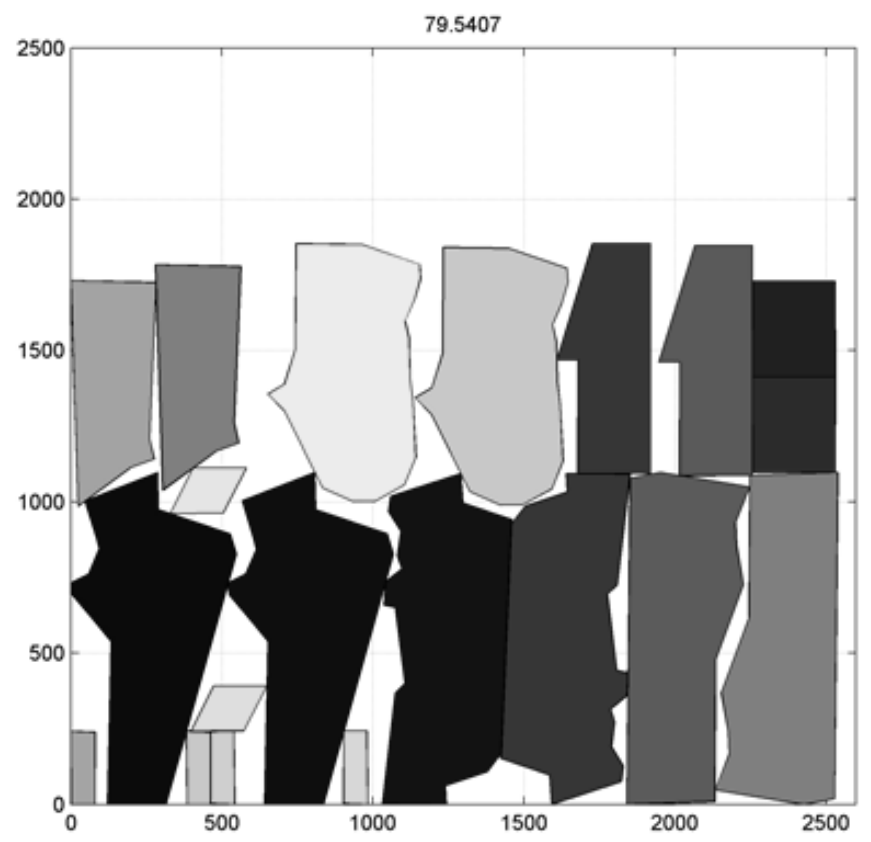

Figure 17. The overall best result for MAO

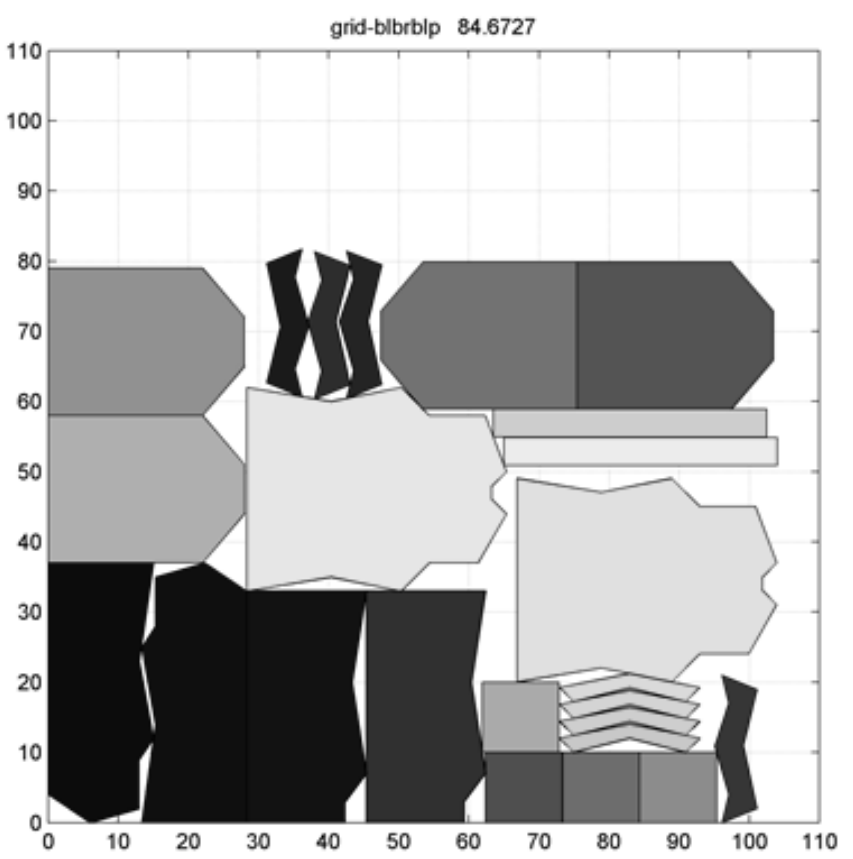

Figure 18. The overall best result for MARQUES 
Some preliminary research has already been conducted on the leather nesting problem, where both the leather and the cutting parts are irregular. Figure 20.a shows leather, and Figure 20.b shows leather in vector format with defined low-quality areas. By applying Grid heuristic on leather (Figure 20.c), overlapping of cutting parts cannot occur. Damaged areas can also be determined and defined as infeasible areas (Figure 20.d). A search direction for a feasible grid point can also be defined, based on material quality. In Figures 20.c and 20.d, a search direction from the center is used. A random search direction can also be defined.

Implemented algorithms can also be applied on material with patterns. To obtain a marker with correct pattern matching, matching points must be defined on a cutting part and placed on a feasible grid point, while the grid density corresponds to the pattern width.

Additional research will be performed to determine the influence of the grid density on marker efficiency.

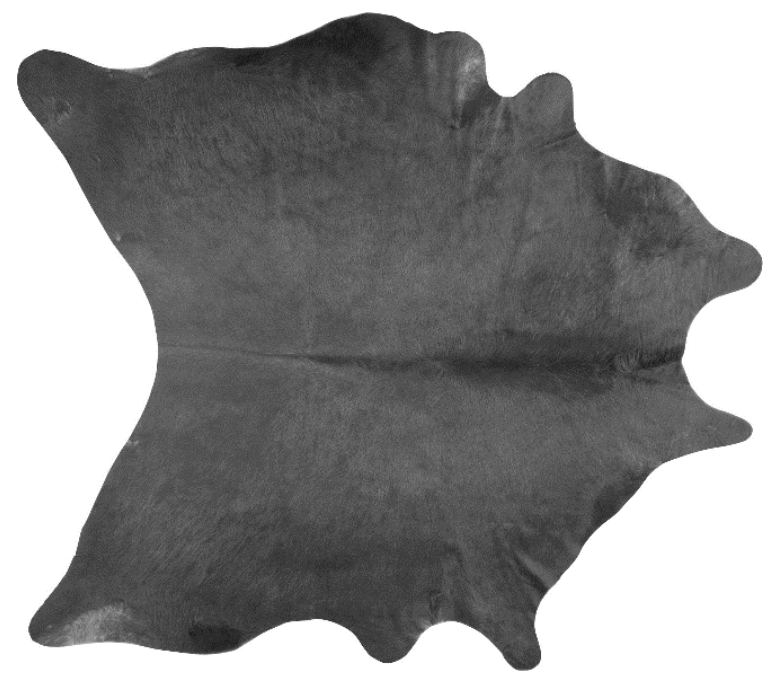

a)

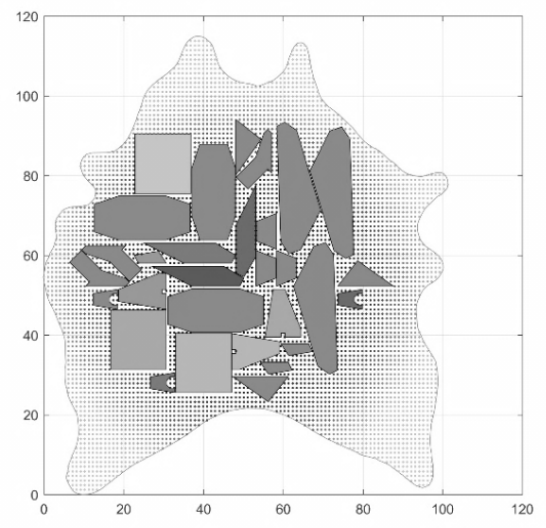

c)

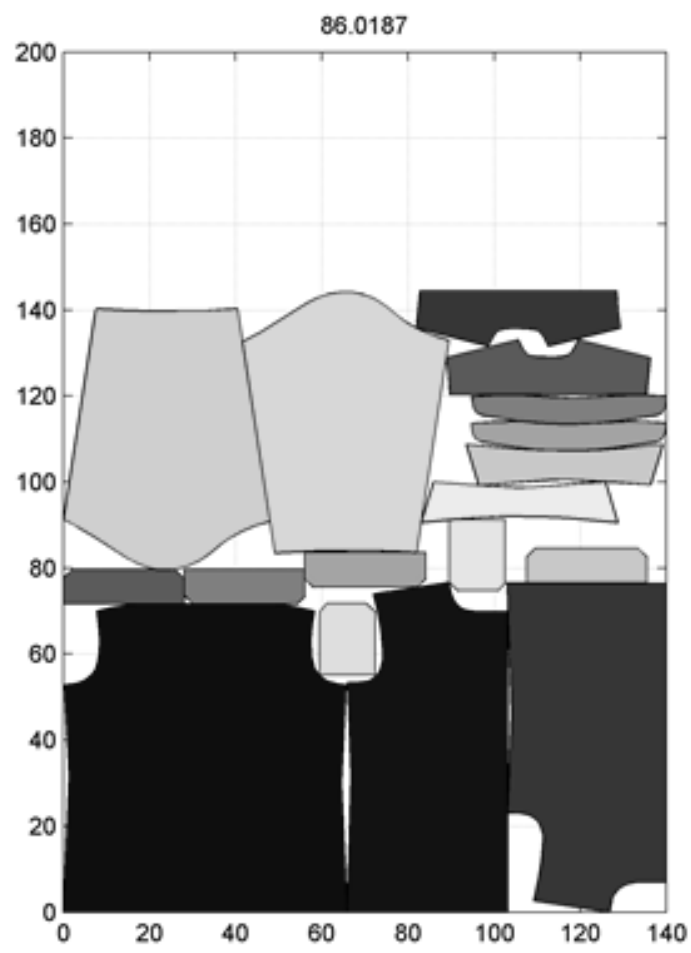

Figure 19. The overall best result for MAN SHIRT

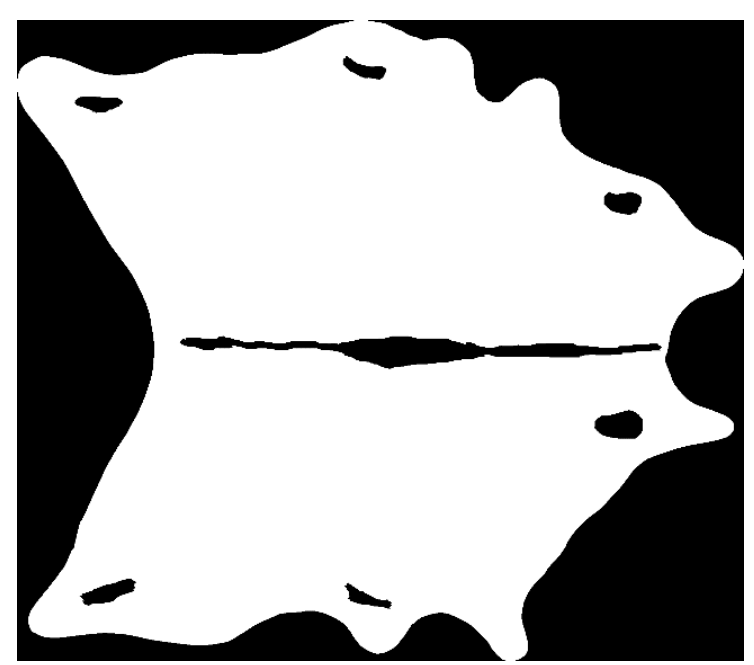

b)

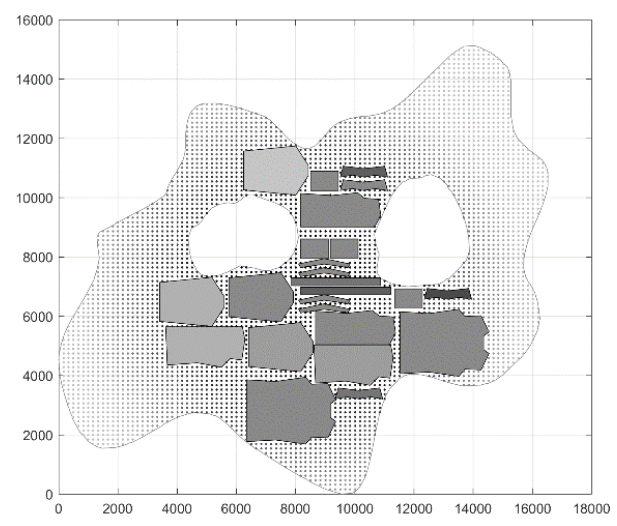

d)

Figure 20. Application of grid heuristics on leather nesting 


\section{Conclusions}

In this research, a problem of marker making has been solved. The purpose of this research was to develop novel heuristics for obtaining a marker to optimize the garment production in terms of improving material utilization, and therefore, influence the production productivity. In this paper, novel heuristics were introduced: Grid, Grid-BLP, Grid-Shaking, and a hyperheuristic approach Grid-all. A novel placement sequence called AEF - All Equal First was introduced to place all equal cutting parts on the marker first, before proceeding to the equal cutting parts of the other group. Methods were hybridized with genetic algorithm. A novel individual representation of GA is designed that is composed of 3 different elements: ordering part, rotation part and a hyper-heuristic part, where a placement heuristic is chosen.

A research has been conducted to determine the efficiency of hyper-heuristic placement and to determine which combination of Grid heuristic creates the best efficiency marker. It was shown that Grid-BLP and Grid-Shaking obtained higher marker efficiency than the Grid heuristics, with Grid-Shaking obtaining the best results for most datasets since it can escape local optima. The hyper-heuristic chose Grid-Shaking in most cases $(88 \%)$, while Grid heuristic was never chosen.

The created algorithm can easily be applied on datasets with markers and cutting parts of arbitrary size and possible damaged areas. Implemented algorithms can also be applied on material with patterns.

\section{Acknowledgements}

This work was fully supported by Croatian Science Foundation under the project number 3011 Application of mathematical modeling and intelligent algorithms in clothing construction.

\section{References}

[1] Dumishllari, E., \& Guxho, G. (2016). Influence of Lay Plan Solution in Fabric Efficiency and Consume in Cutting Section. Autex Research Journal, 16(4). doi:10.1515/aut2015-0055

[2] Yesilpinar, S., \& Aytac, V. (2009). An Approach Aimed at Fabric Consumption in Shirt Production. Textile Research Journal, 79(5), 461-467. doi:10.1177/0040517508090491

[3] Ujević, D., Rogale, D., \& Hrastinski, M. (2010). Tehnike konstruiranja $i$ modeliranja odjeće, treće dopunjeno $i$ prošireno izdanje. Zagreb: Zrinski d. $d$.

[4] Guo, Z., Wong, W., Leung, S., \& Li, M. (2011). Applications of artificial intelligence in the apparel industry: a review. Textile Research Journal, 81(18), 1871-1892. doi:10.1177/0040517511411968

[5] Wäscher, G., Haußner, H., \& Schumann, H. (2007). An improved typology of cutting and packing problems. European Journal of Operational Research, 183(3), 11091130. doi:10.1016/j.ejor.2005.12.047
[6] Egeblad, J. (2008). Heuristics for Multidimensional Packing Problems. Københavns Universitet, Faculty of Science, Datalogisk Institut, Department of Computer Science. Retrieved from http://forskningsbasen.deff. $d k /$ Share.external? sp=S659a8b80-ac03-11de-bc73000ea68e967b\&sp=Sku

[7] Ramesh Babu, A., \& Ramesh Babu, N. (2001). A generic approach for nesting of 2-D parts in 2-D sheets using genetic and heuristic algorithms. Computer-Aided Design, 33(12), 879-891.

[8] Segenreich, S. A., \& Braga, L. M. P. F. (1986). Optimal nesting of general plane figures: A Monte Carlo heuristical approach. Computers \& Graphics, 10(3), 229-237. doi:http://dx.doi.org/10.1016/0097-8493(86)90007-5

[9] Bennell, J. A., \& Oliveira, J. F. (2008). The geometry of nesting problems: A tutorial. European Journal of Operational Research, 184(2), 397-415. doi:10.1016/j. ejor.2006.11.038

[10] Mahadevan, Anantharam. (n.d.). Optimization in Computeraided Pattern Packing (Marking, Envelopes) (1984). North Carolina State University.

[11] Sato, A. K., Martins, T. C., \& Tsuzuki, M. S. G. (2012). An algorithm for the strip packing problem using collision free region and exact fitting placement. Computer-Aided Design, 44(8), 766-777. doi:10.1016/j.cad.2012.03.004

[12] Ghosh, P. K. (1991). An algebra of polygons through the notion of negative shapes. CVGIP: Image Understanding, 54(1), 119-144.

[13] Fujita, K., Akagi, S., \& Hirokawa, N. (1993). Hybrid approach for optimal nesting using a genetic algorithm and a local minimization algorithm. In Proceedings of the 19th annual ASME design automation conference (Vol. 1, pp. 477-484). Retrieved from http://www.waka.kindai.ac.jp/ tea/hirokawa/hirokawa/research/papers/1993/09_ASME_ DAC_nesting.pdf

[14] Hopper, E., \& Turton, B. (1999). A genetic algorithm for a $2 D$ industrial packing problem. Computers \& Industrial Engineering, 37(1), 375-378.

[15] Burke, E., Hellier, R., Kendall, G., \& Whitwell, G. (2006). A New Bottom-Left-Fill Heuristic Algorithm for the TwoDimensional Irregular Packing Problem. Operations Research, 54(3), 587-601. doi:10.1287/opre.1060.0293

[16] Dowsland, K. A., Vaid, S., \& Dowsland, W. B. (2002). An algorithm for polygon placement using a bottom-left strategy. European Journal of Operational Research, 141(2), 371-381.

[17] Jylänki, J. (2010). A thousand ways to pack the bin-a practical approach to two-dimensional rectangle bin packing. retrived from http://clb. demon. fi/files/ RectangleBinPack. pdf. Retrieved from http://clb.demon.fi/ files/RectangleBinPack.pdf

[18] Shalaby, M. A. (2013). A Particle Swarm Optimization Algorithm for a 2-D Irregular Strip Packing Problem. American Journal of Operations Research, 03(02), 268278. doi:10.4236/ajor.2013.32024

[19] Burke, E., \& Kendall, G. (1999). Applying ant algorithms and the no fit polygon to the nesting problem. In Advanced Topics in Artificial Intelligence (pp. 453464). Springer. Retrieved from http://link.springer.com/ chapter/10.1007/3-540-46695-9_38 
[20] Fischetti, M., \& Luzzi, I. (2009). Mixed-integer programming models for nesting problems. Journal of Heuristics, 15(3), 201-226.

[21] Burke, E. K., Gendreau, M., Hyde, M., Kendall, G., Ochoa, G., Özcan, E., \& Qu, R. (2013). Hyper-heuristics: a survey of the state of the art. Journal of the Operational Research Society, 64(12), 1695-1724. doi:10.1057/jors.2013.71

[22] Burke, E. K., Hyde, M., Kendall, G., Ochoa, G., Özcan, E., \& Woodward, J. R. (2010). A Classification of Hyperheuristic Approaches. In M. Gendreau \& J.-Y. Potvin (Eds.), Handbook of Metaheuristics (Vol. 146, pp. 449468). Boston, MA: Springer US. doi:10.1007/978-1-44191665-5_15

[23] Garrido, P., \& Riff, M. C. (2007). Collaboration Between Hyperheuristics to Solve Strip-Packing Problems. In P. Melin, O. Castillo, L. T. Aguilar, J. Kacprzyk, \& W. Pedrycz (Eds.), Foundations of Fuzzy Logic and Soft Computing (pp. 698-707). Springer Berlin Heidelberg.

[24]Bhanu, S. M. S., \& Gopalan, N. P. (2008). A HyperHeuristic Approach for Efficient Resource Scheduling in Grid. International Journal of Computers Communications \& Control, 3(3), 249. doi:10.15837/ijccc.2008.3.2393

[25] Terashima-Marín, H., Farías Zárate, C. J., Ross, P., \& Valenzuela-Rendón, M. (2006). A GA-based method to produce generalized hyper-heuristics for the 2D-regular cutting stock problem (p. 591). ACM Press. doi:10.1145/1143997.1144102

[26] EURO Special Interest Group on Cutting and Packing. (2015). EURO Special Interest Group on Cutting and Packing. Retrieved January 29, 2015, from https://paginas. fe.up.pt/ esicup/

[27] Domovic, D., \& Rolich, T. (2015). Solving strippacking problem using sequence pair. doi:10.1109/ MIPRO.2015.7160455

[28] Domović, D., Rolich, T., Grundler, D., \& Bogović, S. (n.d.). Algorithms for $2 D$ Nesting Problem Based on the No-Fit Polygon. In Proceedings of the 37th International Convention MIPRO 2014: CIS - Intelligent Systems (pp. 1344-1349). Presented at the 2014 37th International Convention on Information and Communication Technology, Electronics and Microelectronics (MIPRO), Opatija, Croatia: Croatian Society for Information and Communication Technology, Electronics and Microelectronics - MIPRO.

[29] Toledo, F. M. B., Carravilla, M. A., Ribeiro, C., Oliveira, J. F., \& Gomes, A. M. (2013). The Dotted-Board Model: A new MIP model for nesting irregular shapes. International Journal of Production Economics, 145(2), 478-487. doi:10.1016/j.ijpe.2013.04.009

[30] Chazelle, B. (1983). The Bottom-Left Bin-Packing Heuristics: An Efficient Implementation. IEEE Transactions on Computers, c-32(8), 697-707.

[31] Jakobs, S. (1996). On genetic algorithms for the packing of polygons. European Journal of Operational Research, 88(1), 165-181.
[32] Hopper, E. (2000). Two-dimensional packing utilising evolutionary algorithms and other meta-heuristic methods (Doctoral Thesis). University of Wales. Cardiff. Retrieved from http://vmk.ugatu.ac.ru/c\%26p/article/HOPPER/ PhDisser/part1.pdf

[33] Golub, M. (2004). Genetski algoritam - prvi dio. Retrieved February 24, 2014, from http://www.zemris.fer.hr/ golub/ ga/ga_skripta1.pdf

[34] Dasgupta, S., Papadimitriou, C. H., \& Vazirani, U. V. (2006). Algorithms. Retrieved from http://www.cse.ucsd. edu/ dasgupta/mcgrawhill/

[35] Pohlheim, H. (2007, March). GEATbx - Genetic and Evolutionary Algorithms Toolbox in Matlab. GEATbx - The Genetic and Evolutionary Algorithm Toolbox for Matlab. Retrieved February 21, 2014, from http://www.geatbx.com/

[36] Pohlheim, H. (1998). Genetic and Evolutionary Algorithm Toolbox for use with MATLAB. Dept. Comput. Sci., Univ. Ilmenau, IImenau, Germany. Retrieved from http://www. geatbx.com/download/GEATbx_Tutorial_v33c.pdf

[37] Factorial of input - MATLAB factorial. (n.d.). Retrieved December 6, 2016, from https://www.mathworks.com/ help/matlab/ref/factorial.html? requestedDomain $=w w w$. mathworks.com

[38] Knuth, D. E. (1997). The Art of Computer Programming, Volume 2 (3rd Ed.): Seminumerical Algorithms. Boston, MA, USA: Addison-Wesley Longman Publishing Co., Inc.

[39] Knuth, D. E. (1998). The Art of Computer Programming, Volume 3: (2Nd Ed.) Sorting and Searching. Redwood City, CA, USA: Addison Wesley Longman Publishing Co., Inc.

[40] Pohlheim, H. (2003). Evolutionary Algorithms. Springer, Heidelberg. Retrieved from http://www.geatbx.com/ download/GEATbx_Intro_Algorithmen_v37.pdf

[41] Schlierkamp-Voosen, D., \& Mühlenbein, H. (1993). Predictive models for the breeder genetic algorithm. Evolutionary Computation, 1(1), 25-49.

[42] Egeblad, J., Nielsen, B. K., \& Odgaard, A. (2007). Fast neighborhood search for two- and three-dimensional nesting problems. European Journal of Operational Research, 183(3), 1249-1266. doi:http://dx.doi. org/10.1016/j.ejor.2005.11.063

[43] Bennell, J. A., \& Song, X. (2010). A beam search implementation for the irregular shape packing problem. Journal of Heuristics, 16(2), 167-188. doi:10.1007/s10732008-9095- $x$

[44] Optitex. (2017). Retrieved February 27, 2017, from http:// optitex.com/

[45] NestLib. (2018). Retrieved June 2, 2018, from https:// nestlib.geometricglobal.com/ 